Precarización y formas de supervivencia en la sociedad portuguesa - de proletarios agrícolas a desempleados y mal empleados

Inês Fonseca

páginas / año $9-n^{\circ} 21$ Septiembre-Diciembre / ISSN 1851-992X/ pp. 34-47/

2017

http://revistapaginas.unr.edu.ar/index.php/RevPaginas

\title{
Precarización y formas de supervivencia en la sociedad portuguesa - de proletarios agrícolas a desempleados y mal empleados
}

\section{Precarization and forms of survival in Portuguese society - from agricultural proletarians to unemployed and poorly employed}

\author{
Inês Fonseca \\ Faculdade de Ciências Sociais e Humanas \\ da Universidade NOVA de Lisboa, Portugal \\ pintofonseca.ines@gmail.com
}

\section{Resumen}

El presente texto constituye una reflexión sobre el fenómeno de precarización en la sociedad portuguesa y la situación actual de la población de una localidad de los alrededores de Lisboa que, desde los años 80, atraviesa un proceso de desindustrialización -tema de una investigación sobre los efectos de estos fenómenos en la organización y en las estrategias familiares y redes sociales.

\section{Palabras Clave}

Precarización; supervivencia; sociedad portuguesa; proletarios agrícolas; desempleados; mal empleados

\begin{abstract}
This text is a reflection on the phenomenon of precarization in Portuguese society and the current situation of the population of a locality around Lisbon that, since the 80s, is going through a process of deindustrialization - a topic of research on the effects of these phenomena in the organization and in family strategies and social networks.
\end{abstract}

\section{Keywords}

Precarization; survival; Portuguese society; agricultural proletarians; unemployed; poorly employed 


\section{Introducción}

La transformación de la economía mundial, en el último cuarto del siglo XX, produjo importantes transformaciones en el mundo del trabajo. En Europa, la desindustrialización (derivada, en parte, del progreso tecnológico) acompañó la terciarización del tejido productivo y la deslocalización de la producción. En las llamadas economías emergentes, se observó un proceso de industrialización y el surgimiento de nuevas clases obreras. Por todas partes se instaló la flexibilización de la economía y los mercados de trabajo.

Simultáneamente, en los discursos políticos y de los medios de comunicación social, empiezan a surgir referencias recurrentes a las situaciones de pobreza / exclusión / precariedad de los individuos y los pobres / excluidos / trabajadores precarios pasan a ser objeto de la atención y las buenas intenciones de todos. La preocupación con el desempleo y la precariedad pasa a formar parte de la vida cotidiana de los ciudadanos.

Como estas categorías remiten a diferentes perspectivas y análisis teóricos que convergen sobre el mismo fenómeno, se impone una clarificación. ¿A qué nos referimos al hablar de precarización social?

\subsection{Precarización social}

La emergencia de la temática de la precariedad en las ciencias sociales surge, precisamente, en el momento en que progresa el crecimiento de las tasas de desempleo y se imponen formas atípicas de empleo a consecuencia de las condiciones de producción en las sociedades contemporáneas.

En las últimas décadas (en los países de Europa) hemos asistido al cuestionamiento del modelo de estabilidad que significaba la existencia de "un empleo de por vida", con contrato fijo y a tiempo completo, en empresas que incentivaban a los trabajadores a planificar largas carreras en su seno. Progresivamente, a esta situación que permitía la aspiración a una movilidad social ascendente a la mayoría de los trabajadores, se le contrapone otra caracterizada por la exigencia creciente de flexibilidad y movilidad a los trabajadores, cada vez menos protegidos en sus derechos sociales que proceden de la condición salarial.

La situación descrita podría hacer que nos dejáramos seducir por la imagen de un mundo del trabajo dividido y que funciona a dos velocidades: el centro (estabilizado) y los márgenes (inestables) -que reflejaría la existencia de dos condiciones diferentes en relación a la posición de los trabajadores en el mercado de trabajo. Por un lado, un grupo de trabajadores integrados en la condición salarial (cubiertos por un contrato de trabajo a tiempo indeterminado y los derechos sociales inherentes) y, por otro, un grupo de trabajadores marginales cuya situación laboral es precaria (con contratos de trabajo de media o corta duración y sin los derechos sociales de la condición de trabajador asalariado). 


\section{Precarización y formas de supervivencia en la sociedad portuguesa - de proletarios agrícolas a desempleados y mal empleados}

No obstante, si con frecuencia la precariedad laboral obliga a los individuos a someterse a situaciones al margen de la condición salarial, este fenómeno no puede ser considerado como exterior a la propia sociedad salarial.

En realidad, desde el punto de vista de las entidades patronales, la precariedad de los trabajadores (o de una parte de estos) resulta complementaria a la condición laboral estable. Si ambas situaciones generan condiciones diversas, ellas coexisten y son interdependientes, constituyendo las dos caras de una misma moneda, que es la gestión de la mano de obra y de los mercados de trabajo. A través de dispositivos existentes, con matices, en los diferentes códigos del trabajo nacionales (que establecen y regulan el recurso a empleos atípicos), es posible: por un lado, gestionar los flujos de efectivos necesarios para las empresas en función de los objetivos de rentabilidad y, por otro, practicar políticas de bajos salarios y obtener los niveles de productividad deseados (al introducir un elemento de competencia entre los trabajadores -estables y precarios- en la lucha por un empleo).

En este sentido, nos parece de gran utilidad el concepto de precarización social que ha venido siendo desarrollado por Béatrice Appay y Annie Thébaud-Mony 1 , centrado en la cuestión del proceso de creación de la precariedad como fenómeno estructural y que analiza la transformación de los modos de producción y de organización del trabajo que contribuyen a la emergencia del fenómeno.

Se trata, de un proceso social que afecta a la vida de las personas y no de un estado o de una etapa de vida - lo que remitiría a una naturalización de la situación de precariedad que afecta a los individuos y que les atribuiría la responsabilidad por esa misma situación².

\subsection{Salariado y precariado}

En la actualidad, la precariedad existente en relación al tipo de empleo (dependiendo de las formas de contratación) y a las condiciones de trabajo, implica para determinados trabajadores la existencia de ingresos inciertos con perspectivas de vida a corto plazo, al tiempo que coloca a los trabajadores titulares de contratos fijos (regulados por convenios colectivos de trabajo) bajo la amenaza, cada vez más plausible, de poder ser despedidos de un momento a otro.

Por más que el salariado continúe siendo la situación dominante entre la población activa y que las situaciones atípicas de empleo permanezcan minoritarias,

\footnotetext{
${ }^{1}$ El concepto fue debatido en profundidad en los trabajos llevados a cabo en el marco de la "Action scientifique fédérative de l'IRESCO", que adoptó el trabajo y la salud como indicadores del proceso de precarización social, y dio como resultado la publicación colectiva titulada "Précarisation sociale; travail et santé", bajo la orientación de ambas autoras. Este análisis, que surgió de la observación de variables que indicaban una aceleración y una generalización de la precarización económica, suscitando la necesidad de investigar acerca de sus efectos directos en la vida (laboral y familiar) de los individuos, ha tenido continuidad en los respectivos trabajos posteriores.

${ }^{2}$ Béatrice Appay et al., Précarisation salariale et précarisation familiale - rapport finale. París, CNAFCNRS, 1999
} 


\section{Inês Fonseca}

se ha instalado un sentimiento generalizado de inseguridad y de inestabilidad en relación al futuro. En este sentido, el aumento de la precariedad no representa el fin progresivo de la condición salarial, sino antes su transformación: la existencia de más trabajadores precarios contribuye al aumento del fenómeno del sentimiento de inseguridad de los trabajadores con un empleo estable y a la existencia de una mano de obra dócil y disponible para aceptar condiciones de trabajo cada vez peores.

En el análisis de este fenómeno es fundamental tener en cuenta el hecho de que éste constituye una consecuencia de la organización capitalista contemporánea, en la medida en que para las entidades patronales se trata de hacer recaer sobre los propios trabajadores los riesgos asociados al puesto de trabajo, externalizando las protecciones sociales, así como las garantías de protección en caso de pérdida de empleo (seguros de desempleo). Los vínculos entre los asalariados y las entidades patronales se hacen más frágiles, pasando a ser los propios trabajadores quienes deban asumir los riesgos asociados, una vez que todo este proceso, a nivel del mercado de trabajo, viene acompañado por un proceso correspondiente de desinversión en el marco de protección a los trabajadores asumido colectivamente por la sociedad 3 .

Diferentes autores apuntan a una generalización de las condiciones de trabajo precarias, con tendencia a convertirse en estructurales, para las cuales prevén un "bello futuro" 4. Esta perspectiva sobre la actualidad del mundo del trabajo sitúa su problematización en el centro del debate sobre la cuestión social, el Estado de bienestar y los derechos sociales del salariado. Simultáneamente, surge como central la problemática de la transformación del valor del trabajo y de las prácticas laborales de las clases trabajadoras, diferentes en función de que la condición laboral sea estable o precaria.

\section{El "otro" en situación de precariedad o desempleado}

A pesar de las profundas transformaciones sociales que se están produciendo a consecuencia de las mutaciones en el mundo del trabajo, esta temática ha sido descuidada por el análisis antropológico ${ }^{5}$. Una explicación posible para esta situación podrá ser el hecho de parecer existir entre una parte de los investigadores de la disciplina una adhesión total a las teorías sobre el fin del trabajo y de la clase

\footnotetext{
3 En este sentido, la obra de Robert Castel (2003) constituye un hito importante en el análisis de la constitución de la condición salarial y el posterior "effritement de la condition salariale" (en las palabras de este autor). Este proceso consiste en una "diversification des formes de contrat de travail qui signifient pour les salariés une perte des protections et droits attachés au travail” (Beaud y Cartier, 2006: 567) y trae como consecuencia una segmentación de los asalariados.

4 Beaud, Stéphane y Cartier, Marie, “De la précarisation de l'emploi à la précarisation du travail. La nouvelle condition salariale”; in: S. Beaud et al., La France invisible. París, La Découverte, 2006, 5 La mayor parte de los estudios sobre el reciente fenómeno de la inmigración hacia los países occidentales se ocupan, sobre todo, del tema de las identidades culturales y la reconstrucción de identidades, ignorando por completo la cuestión del trabajo (que siempre es el motivo principal del desplazamiento de estas personas) Los inmigrantes son vistos por los antropólogos como minorías étnicas o culturales, y raramente como trabajadores.
} 


\section{Precarización y formas de supervivencia en la sociedad portuguesa - de proletarios agrícolas a desempleados y mal empleados}

obrera. Con todo, la realidad nos muestra que siguen existiendo trabajadores: únicamente han cambiado sus condiciones de existencia conforme las formas de su explotación han evolucionado 6 .

La cuestión que actualmente tiene sentido plantear es la siguiente: en un contexto mundial de generalización del desempleo y del trabajo precario, ¿qué pasa a nivel de la construcción y reproducción de las identidades de los trabajadores? El conocimiento producido por la antropología sobre otras situaciones, permitiendo comparaciones con la actual realidad vivida por los trabajadores, constituye un importante patrimonio a utilizar. Especialmente, los análisis producidos sobre la construcción de identidades de los trabajadores de los más variados sectores, sobre la cultura obrera (su construcción y reproducción), sobre las relaciones de dominación y subordinación establecidas en contextos laborales, etc.

Un rasgo común de las investigaciones antropológicas (aquellas que, un poco a contracorriente, se van realizando) sobre el mundo del trabajo, consiste en la afirmación de la diversidad del sentido del trabajo ${ }^{7}$. De hecho, ésta es, a nuestro entender, la principal contribución de la disciplina para el análisis de lo que pasa actualmente en el mundo laboral, la idea de que la relación de los individuos con el trabajo está íntimamente asociada a una comunidad de pertenencia y a unos valores compartidos (entre los cuales se incluye el del trabajo). A través de categorías socialmente construidas (como trabajo o trabajadores, desempleo o desempleados), los individuos construyen procesos de identificación, se clasifican a sí mismos y a los demás y son clasificados por otros.

El significado atribuido al trabajo por los científicos sociales, por los agentes de la administración pública (local o central) o por otros grupos de la sociedad podrá ser (y es, muchas veces) distinto del significado dominante entre los individuos de un determinado grupo en estudio u objeto de la intervención de las políticas públicas.

En muchas de las investigaciones que se ocupan del mundo del trabajo es perceptible la influencia de la orientación teórica propuesta por Edward P. Thompson en su obra sobre la formación de la clase obrera inglesa, en que el autor recuerda que su constitución es, sobre todo, el resultado de un largo proceso de construcción social, con avances y retrocesos, con fases que se superponen las unas a las otras, que permanecen y coexisten y que, por encima de todo, difícilmente se trata de un proceso definitivo o de una condición inmutable.

\footnotetext{
${ }^{6}$ Kasmir, Sharryn y Carbonella, August, "Dispossession and the anthropology of labor"; in: Critique of Anthropology, 28 (5), pp. 5-25, 2008. Narotsky, Susana, "The political economy of affects: community, friendship, and family in the organization of a spanish economic región"; in: A. Procoli [dir.], Workers and narratives of survival in Europe. The management of precariousness and the end of the twentieth century, Albany, State University of New York Press, 2004, pp. 57-79.

7 Procoli, Angela [dir.], Workers and narratives of survival in Europe. The management of precariousness and the end of the twentieth century. Albany, State University of New York Press, 2004
} 


\section{De los campos a la fábrica y de las industrias a las superficies comerciales}

A pesar de asumir algunos elementos innovadores, el proceso de generalización de la precariedad (ya identificado como un modelo de gestión de la mano de obra) ${ }^{8}$ constituye una práctica antigua por parte de las entidades patronales. Como ha sido referido por Thompson y Castel, la historia de la clase obrera se cuenta a través de múltiples situaciones de precariedad y está repleta de luchas (por mejores condiciones de trabajo, mejores salarios y más derechos sociales) para regular la condición salarial (que constituye el modelo ideal). La utilización de esta forma de gestión de la mano de obra a través del mantenimiento en situación precaria de una parte de los trabajadores asalariados nunca ha dejado de existir, se mantiene y asume nuevos formatos a lo largo del tiempo?.

\subsection{El caso portugués}

En Portugal, debido al atraso del desarrollo industrial, no sólo se produjo una estabilización tardía de la clase obrera (inicialmente circunscrita a pequeños núcleos de trabajadores empleados en la industria del vidrio, del corcho, tipográfica, etc.) como también el proceso de desmantelamiento de esta condición tuvo inicio relativamente antes. Si, de un lado, durante gran parte del siglo XX (1926-1974), el mercado de trabajo portugués obedeció a la regulación de inspiración corporativa y esencialmente paternalista, por parte del estado autoritario; de otro, a partir de 1986 (fecha de la entrada de Portugal en la Comunidad Económica Europea) tuvo inicio la "modernización" del mercado laboral y el progresivo desmantelamiento de la condición salarial estable.

El país constituye, así, un ejemplo particular en la aplicación y evolución de las políticas laborales, caracterizado por mantener un mercado laboral flexible y una población trabajadora en situación de inestabilidad por lo que se refiere a sus derechos laborales y sociales. En este contexto, Baixa da Banheira, por el origen rural de su población (mayoritariamente formada por proletarios agrícolas y pequeños campesinos pobres), supone un caso especialmente interesante en la observación de este fenómeno.

\footnotetext{
${ }^{8}$ El fenómeno que se produce actualmente se traduce en una generalización sistemática de las situaciones de desempleo y de subempleo a todos los sectores del mercado de trabajo y en la posibilidad de que estas vengan a hacerse dominantes y (sobre todo en los países occidentales desarrollados) se extiendan a todas las clases sociales. En este sentido, es bastante justo el comentario de Evelyn Perrin (2006), sobre el contexto laboral francés actual, cuando recuerda que se trata de un contexto de desmantelamiento de derechos sociales existentes $\mathrm{y}$, por lo tanto, de una situación de degradación de la condición salarial que significa, para unos, la pérdida de derechos adquiridos y, para otros, la imposibilidad de cualquier forma de movilidad ascendente e incluso de simple estabilidad laboral.

${ }_{9}^{9}$ Cingolani, Patrick, La Précarité. París, PUF, 2006
} 


\section{Precarización y formas de supervivencia en la sociedad portuguesa - de proletarios agrícolas a desempleados y mal empleados}

\subsection{La población de Baixa da Banheira}

Integrada en la Región Metropolitana de Lisboa, Baixa da Banheira se localiza en el otrora llamado "cinturón industrial" de Lisboa, en la margen sur del río Tajo (que incluye diversos municipios como Alcochete, Almada, Barreiro, Moita, Montijo, Seixal, etc.).

Toda esta zona conoció, desde la década de 40, un fuerte crecimiento demográfico. Una gran cantidad de mano de obra, procedente sobre todo del sur del país (de las regiones rurales y pesqueras de Alentejo y Algarve), aunque también de las regiones del norte interior del país (Trás-os-Montes y Beira-Baixa), acudió a trabajar a una región que se industrializaba (con la instalación de varias unidades de preparación y transformación de corcho, la construcción de las líneas de ferrocarril del sur y sureste -que transformaron a Barreiro en el eje central de las comunicaciones norte-sur del país- y la instalación de los talleres generales de la empresa ferroviaria). Hasta entonces, las principales actividades desarrolladas estaban relacionadas con el sector de la pesca y de la industria salinera.

El crecimiento económico y urbano de este cinturón industrial se produjo especialmente a partir de los años 60, cuando surgen o se consolidan nuevas industrias: agroquímica (CUF), astilleros navales (Lisnave y Setenave), siderurgia (Siderurgia Nacional) y diversas fábricas textiles (que ocupaban mayoritariamente la población femenina). Con la instalación de una mano de obra recién llegada, atraída por la disponibilidad de trabajo, Baixa da Banheira se transforma en una barriada dormitorio ${ }^{10}$. Este contingente se sumó a la población obrera industrial, con el objetivo de alcanzar estabilidad de empleo, mejores salarios y más derechos sociales (algo que el régimen latifundista, dominante en el sur del país, nunca les había ofrecido).

En las tres últimas décadas, toda la región pasa por un fenómeno de desactivación industrial y de crecimiento del sector terciario (con la llegada masiva de las grandes superficies comerciales), al tiempo que en el sector secundario, que todavía existe en pequeña escala, destacan las empresas de pequeña y media dimensión (en el sector de la construcción civil y de la producción alimentaria y agroalimentaria). Para comprender la dimensión de la transformación económica en curso es significativo el hecho de que las mismas instalaciones que otrora albergaron unidades de producción industriales, acogen ahora los hipermercados.

Esta modificación del tejido productivo de la región, caracterizada por un trasvase del sector secundario al terciario, se acompaña no sólo de un crecimiento de la tasa de desempleo (consiguiente a la disminución de los puestos de trabajo necesarios a la nueva actividad económica), sino también de una degradación de las condiciones de empleo y de trabajo y de los derechos sociales asociados.

\footnotetext{
10 En aquella época, Baixa da Banheira pertenecía al distrito municipal de Alhos Vedros y era una de las poblaciones del país con mayor densidad de población, lo que la llevó a ser designada "barrio Shangai".
} 
Una gran parte de la población se encuentra en situación de desempleo y de precariedad profesional, económica y social.

\subsection{El regreso a la condición precaria}

Surgen nuevas formas de empleo y de trabajo, que implican otras tantas modalidades de precariedad para esta población que ya había conocido, en la primera generación de emigrantes, una situación similar (en términos de inestabilidad de vida y de inseguridad en relación al futuro). La conciencia de esta situación atraviesa sus discursos, que refieren de forma recurrente el siguiente estribillo: “- Nosotros salimos de nuestra tierra, en busca de una vida mejor, porque no teníamos un trabajo estable. Y ahora, vemos cómo nuestros hijos están en una situación peor que la nuestra".

Esta situación representa un contratiempo en las expectativas de vida construidas por las generaciones anteriores y que pueden ser resumidas a través de la línea progresiva: formación $\rightarrow$ estabilidad profesional / familiar $\rightarrow$ independencia de las generaciones más jóvenes respecto a las anteriores $\rightarrow$ cuidados prestados a las generaciones mayores. En los discursos de los individuos producidos en la actualidad, se insiste en la idea de que se ha producido un retroceso en los derechos sociales alcanzados anteriormente $\mathrm{y}$, en muchas familias, son los miembros pertenecientes a las generaciones mayores (muchos en situación de prejubilación o de desempleo con derecho a subsidio) quienes deben seguir apoyando a las generaciones más jóvenes de la familia (generalmente con empleos precarios y mal remunerados, con frecuentes períodos de desempleo y con problemas en relación a la vivienda, la escolarización / formación profesional, los cuidados de salud, etc.), lo que consideran un retorno a una situación de partida en sus vidas.

\subsection{Las "armas de los débiles"}

El contexto descrito para Baixa da Banheira nos revela una población en una encrucijada: ante la evidente imposibilidad de encontrar un "buen empleo" (estable, con un salario considerado razonable y dotado de los derechos sociales inherentes) y la necesidad de buscar un trabajo que permita la subsistencia económica y/o el deber de cumplir las formalidades de la demanda de un empleo (impuesto por la administración pública). Naturalmente, las personas que se encuentran en este impasse van a desarrollar estrategias para solucionarlo.

Nuestra hipótesis es que esas estrategias de vida constituyen la adaptación de antiguas situaciones ya vividas y, en ese sentido, se cuentan entre ellas la 


\section{Precarización y formas de supervivencia en la sociedad portuguesa - de proletarios agrícolas a desempleados y mal empleados}

reproducción de una determinada "economía moral"11 y la puesta en práctica de "formas de resistencia cotidiana" 12.

\subsubsection{El retorno de la precariedad y el (casi) regreso al pueblo}

Durante el trabajo de campo que llevé a cabo en la aldea de Aivados (en Baixo Alentejo), tuve oportunidad de conocer a su comunidad de emigrantes residentes en Baixa da Banheira13. La mayoría salió de la aldea en los años 60 , en busca de trabajo. Y recalaron en Baixa da Banheira por el clásico sistema de construcción de redes de trabajadores emigrantes, en que parientes / vecinos / amigos emigrados llaman a otros para que se instalen en la misma región de destino - donde encuentran trabajo.

En 1995, muchas de aquellas familias sufrían las consecuencias del proceso de desindustrialización y de la crisis de desempleo. En la generación de los mayores (aquellos que emigraron de la aldea), muchos hombres habían estado o estaban recibiendo el subsidio de desempleo. Encontrar un nuevo empleo era una tarea difícil por el obstáculo de la edad (cuya media rondaba los 55 años). Las mujeres, por su parte, habían sido las primeras en quedarse sin trabajo, siendo inmediatamente relegadas a la esfera del trabajo doméstico y de las actividades informales (que ya habían desempeñado en el inicio de sus vidas). La generación más joven (los hijos de quienes emigraron), a su vez, se debatía con dificultades en su entrada y permanencia en el mercado de trabajo: saltaban de cursillo de formación en cursillo de formación (de los más variados tipos) y alternaban empleos temporales con períodos de desempleo.

\footnotetext{
11 Thompson, Edward P., Costumbres en común. Barcelona, Crítica, 1995. Esta noción introduce, como refiere el autor, la idea de reposición del equilibrio social: grupos con derechos y deberes, que colocan a unos en relación de subordinación respecto a los otros y en que los dominados detentan también algunos derechos frente a los dominantes. La forma como esos derechos de los dominados son ejercidos depende de las "negociaciones" establecidas entre ambos grupos. Cuando el desequilibrio se instala en estas relaciones, aumentando las desigualdades y haciendo aún más frágil la situación de los dominados, surgen reacciones. Estas pueden estar fuera del marco legal o de la aceptación dominante de la sociedad. El autor estudió la sociedad campesina feudal y sus concepciones de justicia social, derechos y obligaciones, reciprocidades, que se traducían en instituciones sociales inscritas en la vida cotidiana. Nuestra hipótesis es que el funcionamiento del Estado de bienestar, en las sociedades democráticas contemporáneas, genera el mismo tipo de ambiciones y aspiraciones entre los grupos de subordinados. Aunque en un contexto social diferente, también surgen reacciones que buscan el reequilibrio, cuando las instituciones (en este caso, del estado social) no funcionan como se espera.

12 En su obra Weapons of the weak, Scott (1985) parte de la noción de "economía moral" de Thompson y nos muestra cómo grupos dominados hacen sentir su "fuerza" a quienes les dominan, a través de pequeñas acciones de resistencia, que califica de cotidianas. Estas formas de resistencia se integran en la propia actividad social de los individuos y pueden surgir encubiertas en las acciones cotidianas de trabajo, en las relaciones sociales (parentesco, amistad, vecindad), etc.. No se trata, por lo tanto, de alterar un orden social establecido, como de acciones defensivas y de supervivencia y que, en el caso en estudio, pueden pasar por el trabajo clandestino (sin la debida declaración de la renta y el correspondiente pago de impuestos y prestaciones sociales) para familiares y amigos $\mathrm{u}$ otros.

${ }^{13}$ Fonseca, Inês, Aivados. Posse da terra, resistência e memoria no Alentejo. Lisboa, Dinossauro, 2006
} 


\section{Inês Fonseca}

Todos estaban empezando a buscar la mejor manera de adaptarse a la situación de crisis, con el regreso al desempleo y al empleo precario en una trayectoria de vida que habían imaginado más estable. En el primer contacto, rápidamente quedó claro que, ante la realidad con que se deparaban, las estrategias seguidas variaban, según los individuos y las familias.

En el caso de una de las familias, inmediatamente fue expresado el deseo de regreso a la tierra natal. Se trataba de una pareja emigrante (con 54 y 58 años), con una hija adulta ya nacida en Baixa da Banheira (recientemente salida de un período de desempleo y de un divorcio) y con dos nietos (la chica, de 13 años, en proceso de abandono escolar precoz, y el chaval, de 8 años).

Único obstáculo al proyecto de regreso al pueblo: proletarios agrícolas que habían sido antes de salir de Alentejo (sin casa ni tierra propia y sin herencia recibida), no tenían un sitio al cual volver. Por eso, la materialización del proyecto tendría que pasar por la compra de un pequeño terreno con una casa, destinando a ese fin el dinero de la indemnización recibida por el despido del marido. Pero la incertidumbre era grande: si, por un lado, el regreso al pueblo les permitiría reducir los gastos cotidianos (pues el coste de la vida es menor allí), por otro, la salida de Baixa da Banheira, donde las perspectivas de empleo (a pesar de escasas) siempre parecían más probables, implicaba una opción arriesgada.

Finalmente, toman la decisión e incluso consiguen algunos resultados en relación al trabajo: ella continúa "haciendo costura para fuera" y él obtiene un empleo en una pequeña industria de extracción de piedra, cerca de Aivados. La nieta, que entre tanto abandonó la escuela, quedó a cargo de los abuelos en la aldea (donde todos consideraron que estaba más protegida).

Sin embargo, esta situación nunca representó un verdadero regreso al pueblo, sino antes una estrategia asumida colectivamente por toda la familia, destinada a prevenir la inestabilidad futura (que era previsible). Si la pareja mayor regresó al pueblo, lo hizo sólo parcialmente: las clientas de los trabajos de costura seguían siendo en su gran mayoría de la zona de Baixa da Banheira, el marido continuaba inscrito en el Centro de Empleo como candidato a un trabajo (acudiendo a todas las entrevistas a las que era convocado) y ambos continuaban utilizando el Centro de Salud de Baixa da Banheira (considerado mejor). Esta situación implicó idas y venidas constantes, sólo posibles gracias al hecho de conservar la casa de Baixa da Banheira, donde se quedó el resto de la familia: la hija (cocinera en un comedor escolar) y el nieto (estudiante en la escuela preparatoria).

Resumiendo, para hacer frente a una situación de adversidad, esta familia dinamizó su red social para comprar una casa en su aldea natal y obtener un empleo para el jefe de familia. Con todo, siempre estuvo presente la idea de que, más tarde o más temprano (cuando terminase el trabajo en la cantera), tendrían que regresar a Baixa da Banheira, con la cual conservaban lazos importantes (la casa y la familia que se había quedado allí, pero también la protección social -en la salud y en la búsqueda de empleo). Por otro lado, recurrieron al trabajo en actividades del sector informal para solucionar el problema de la subsistencia económica, hasta alcanzar 


\section{Precarización y formas de supervivencia en la sociedad portuguesa - de proletarios agrícolas a desempleados y mal empleados}

la edad de la jubilación (pudiendo, de nuevo, obtener unos ingresos mensuales que, aunque reducidos, son seguros).

\subsubsection{La "escuela de la cooperativa"}

En los primeros contactos establecidos en el terreno, conocí a un grupo de mujeres trabajadoras (que ahora ya están jubiladas). Todos los días se encuentran en una cafetería local donde pasan un rato de convivencia. Se fueron conociendo en momentos y lugares distintos, pero se hace inmediatamente perceptible un rasgo común entre ellas, que es esta trayectoria de vida ya descrita. Las referencias al mundo del trabajo son omnipresentes en las conversaciones, no sólo el trabajo como operarias fabriles, sino todos los trabajos que han desempeñado a lo largo de sus vidas.

Estas mujeres se refieren a sus encuentros en la cafetería como "la escuela de la cooperativa" 14 en una comparación con la actividad escolar infantil (que muchas de ellas apenas conocieron), en que existe una obligatoriedad de asistencia diaria y la necesidad de justificar las faltas. De esta forma, asimismo, justifican su ausencia del espacio doméstico, situación ésta aún no demasiado bien aceptada, excepto cuando existe un motivo válido (¡como podría serlo el hecho de acudir a la escuela con otras compañeras!).

No deja de ser curioso que, a pesar de la participación militante de muchas de ellas en el Movimento Democrático de Mulheres (MDM) ${ }^{15}$ de Baixa da Banheira, que fue una importante escuela en la defensa de sus derechos de mujeres trabajadores y que también tiene un espacio de reunión, prefieran ahora encontrarse en el contexto de un grupo igualmente relacionado con su vida de trabajo, pero más informal. A fin de cuentas, también sus actividades laborales dependieron de redes informales de contactos y personas conocidas. Este es un grupo construido a lo largo del tiempo (diferente de un grupo de antiguas obreras de una misma fábrica), a medida de los sucesivos encuentros, simpatías, complicidades y experiencias compartidas.

Antes de la emigración, residían con sus padres o maridos y no tenían un trabajo remunerado. Les cabía entonces desempeñar las múltiples tareas domésticas y también algunas tareas agrícolas, consideradas secundarias en función

\footnotetext{
${ }^{14}$ La cafetería se encuentra en el primer piso de un edificio donde (en los años 50) fue fundada una cooperativa de consumo, que sigue funcionando en la planta baja. De ahí el nombre por el que suele ser designado ("el café de la cooperativa"), posteriormente transpuesto y adaptado a la designación del grupo de convivencia.

15 El MDM nació en 1969, bajo el impulso del Partido Comunista Portugués. En el contexto de la celebración de las elecciones parlamentarias de 1969, se formaron varias comisiones electorales femeninas, organizadas por el partido para canalizar diversas vías de oposición al régimen. Una vez finalizado el período electoral, se decidió crear el MDM, con el objetivo de organizar las luchas de las mujeres trabajadoras (apartadas de la organización partidaria clandestina -predominantemente masculina- y de la organización sindical libre -que a dictadura prohibía). El movimiento desarrolla un trabajo de combate por mejores salarios para las mujeres y contra el aumento del coste de la vida, por la igualdad entre mujeres y hombres, por la defensa de los derechos de las mujeres (igualdad jurídica, derecho al aborto, protección de la maternidad, etc.).
} 


\section{Inês Fonseca}

a los ingresos que aportaban a la unidad familiar y que, por eso, son entendidas como auxiliares y no como un verdadero trabajo asalariado. Cuando vienen a la ciudad, sobre todo las solteras, encuentran trabajo como empleadas domésticas internas en casas particulares, donde reciben un pequeño salario (complementado con la alimentación y el alojamiento). En cuanto a las casadas, se instalaron con sus maridos e hijos y comenzaron por hacer pequeños trabajos en el sector informal (limpiar en casas particulares, cuidar niños, cocinar para fuera en ocasiones extraordinarias como fiestas, realizar trabajos de costura para fuera, etc.), al margen de derechos sociales.

En este sentido, la entrada en las fábricas textiles constituyó un salto cualitativo en sus vidas: pasaron a ganar un salario fijo (y más elevado que hasta entonces). La experiencia como obreras constituyó igualmente un momento importante en la construcción de su independencia en cuanto que mujeres, alcanzando un nuevo estatuto: trabajaban fuera de la esfera doméstica, integraban otros grupos de sociabilidad (con las compañeras de trabajo) y gozaban de los derechos sociales inherentes a la actividad salarial.

No obstante, esta situación duró poco tiempo, pues las fábricas textiles de la región fueron las que primero cerraron. Inmediatamente, estas mujeres quedaron de nuevo limitadas a la esfera doméstica, volviendo a desempeñar los mismos pequeños trabajos y constituyendo una verdadera mano de obra clandestina: si, oficialmente, su estatuto era el de amas de casa que se ocupaban de la familia y de las tareas del hogar, también desempeñaban otros trabajos, por los que eran remuneradas, dinamizando e integrando el sector informal en el desempeño de tareas como niñeras, cocineras, empleadas de limpieza, etc.

De esta forma, si la mayoría de la población de Baixa da Banheira ha vivido una gran parte de sus vidas teniendo que hacer frente a la precariedad -al principio, por su situación de proletarios agrícolas o de pequeños campesinos pobres, y, más tarde, por la situación de desempleo motivada por la desindustrialización de la región (tras un período de industrialización tardía, como ya mencionamos)- son las mujeres quienes constituyen el grupo más afectado por esta situación.

\section{Conclusión}

Salvaguardando las debidas distancias, podríamos considerar que (tal como sugieren Italo Pardo ${ }^{16}$ en relación a las clases populares de la ciudad de Nápoles y Susan Day ${ }^{17}$ respecto a las mujeres prostitutas de la ciudad de Londres) también la población de Baixa da Banheira constituye la mano de obra ideal en el actual contexto del mundo del trabajo: flexible y siempre disponible para la movilidad.

\footnotetext{
16 Procoli, Angela [dir.], Workers and narratives of survival in Europe. The management of precariousness and the end of the twentieth century. Albany, State University of New York Press, 2004. 17 Procoli, Angela [dir.], Workers and narratives of survival in Europe. The management of precariousness and the end of the twentieth century. Albany, State University of New York Press, 2004
} 


\section{Precarización y formas de supervivencia en la sociedad portuguesa - de proletarios agrícolas a desempleados y mal empleados}

Pero, si queremos profundizar el debate, podemos (eso sí) afirmar que la situación de esta población representa un ejemplo de cómo la desregulación de los mercados de trabajo viene no sólo a facilitar la existencia de una mano de obra dócil y disponible, sino que permite también, una interrelación entre el mundo del trabajo y el desarrollo de un sector informal de la economía.

Contrariamente a lo que afirma Appay sobre el sentido positivo de la capacidad movilizadora del proceso de precarización social

ne génère-t-elle pas des transformations au sein des familles, une intensification des échanges et des relations, de nouvelles solidarités, voire de nouvelles formes de résistance? ${ }^{18}$

considerase que esa dinamización y reactivación de las relaciones sociales y de las solidaridades puede, también, tener efectos perversos, y que esa resistencia (en el sentido que le atribuye Scott [1985]) no siempre constituye una transformación a mejor de la sociedad y puede, incluso, implicar la conservación de prácticas antiguas.

El caso de Baixa da Banheira así parece demostrarlo. Para sobrevivir, las personas "hacen lo que pueden" y como pueden para salvar las dificultades del desempleo y la precariedad -lo que puede pasar por el recurso a amplia escala a la economía informal, con todo lo que eso implica (por ejemplo, trabajo familiar o para amigos y vecinos, impago de impuestos y prestaciones sociales, etc.). Investigar las prácticas y las conductas de los individuos, así como las narrativas que estos construyen sobre su vida, en este contexto de adaptación y resistencia, será el objetivo del trabajo a realizar.

\section{Bibliografía}

APPAY, Béatrice y THÉBAUD-MONY, Annie [dirs.], Précarisation sociale, travail et santé. París, CNRS-IRESCO, 1997.

APPAY, Béatrice et al., Précarisation salariale et précarisation familiale - rapport finale. París, CNAF-CNRS, 1999

BEAUD, Stéphane y CARTIER, Marie, "De la précarisation de l'emploi à la précarisation du travail. La nouvelle condition salariale"; in: S. Beaud et al., La France invisible. París, La Découverte, 2006, pp. 561-573

${ }^{18}$ Appay, Béatrice et al., Précarisation salariale et précarisation familiale - rapport finale. París, CNAFCNRS, 1999, pp. 2. 


\section{Inês Fonseca}

CASTEL, Robert, Les métamorphoses de la question sociale. París, Gallimard, 2003

CINGOLANI, Patrick, La Précarité. París, PUF, 2006

FONSECA, Inês, Aivados. Posse da terra, resistência e memoria no Alentejo. Lisboa, Dinossauro, 2006

KASMIR, Sharryn y CARBONELLA, August, "Dispossession and the anthropology of labor"; in: Critique of Anthropology, 28 (5), pp. 5-25, 2008

NAROTSKY, Susana, "The political economy of affects: community, friendship, and family in the organization of a spanish economic región"; in: A. Procoli [dir.], Workers and narratives of survival in Europe. The management of precariousness and the end of the twentieth century, Albany, State University of New York Press, 2004, pp. 57-79

PERRIN, Evelyn, “Du salariat au précariat?”; in: P. Cours-Salies y M. Vakaloulis [dirs.]; Nouvelles luttes de classes (Actuel Marx Confrontation). París, PUF, 2006, pp.127-134

PROCOLI, Angela [dir.], Workers and narratives of survival in Europe. The management of precariousness and the end of the twentieth century. Albany, State University of New York Press, 2004

SCOTT, James, The moral economy of the peasant: rebellion and subsistence in Southeast Asia. New Haven / London, Yale University Press, 1976

SCOTT, James, Weapons of the weak: everyday forms of peasant resistance. New Haven / London, Yale University Press, 1985

THOMPSON, Edward P., La formación de la clase obrera en Inglaterra. Barcelona, Crítica, 1989

THOMPSON, Edward P., Costumbres en común. Barcelona, Crítica, 1995

Recibido: 17/09/2017

Evaluado: $27 / 10 / 2017$

Versión Final: 26/12/2017 